\title{
Controlled deposition of diamond from an acetylene-oxygen combustion flame
}

\author{
J. J. Schermer, J. E. M. Hogenkamp, G. C. J. Otter, G. Janssen, W. J. P. van Enckevort and \\ L. J. Giling \\ Experimental Solid State Physics III, Research Institute for Materials, University of Nijmegen. Toernooiveld, 6525 ED, Nijmegen (Netherlands)
}

(Received August 4, 1992)

\begin{abstract}
An experimental set-up for the deposition of diamond by an acetylene-oxygen combustion flame was developed in which it is possible to control the deposition temperature within $10^{\circ} \mathrm{C}$ during long-term experiments. This degree of accuracy is obtained by cooling the back of the substrate holder with an electronically controlled water injection system and using soldered substrates, ensuring a good thermal contact. With this set-up a large number of experiments were performed with deposition times of $1 \mathrm{~h}$. Special attention was given to the growth of diamond layers with uniform thickness and morphology because these properties are considered essential for future applications. It was found that the deposition temperature, the total gas flow, the composition of the gas phase and the position of the substrate in the flame all have major influences on the deposit. Not only the growth rate and the quality of the diamond are strongly influenced by the deposition conditions, but also the preferred crystal habit and the homogeneity.
\end{abstract}

\section{Introduction}

Since Hirose and Kondo [1] reported their first experiments, the use of an acetylene-oxygen torch for diamond synthesis has become a common technique. Despite the simplicity and low cost of the experimental set-up, good quality polycrystalline diamond films [2] can be achieved with high growth rates. Since then several authors have investigated the influence of the substrate temperature and the oxygen-to-acetylene flow ratio on flame grown diamond [2-4], while the radial inhomogeneity of diamond deposits has been studied by Oakes et al. [5] using deposition runs of $10 \mathrm{~min}$. The main problem in the flame process is not to measure the actual deposition temperature, but to maintain accurate temperature control during extended growth experiments. Therefore, a new temperature control system was developed based on a similar principle to that described by Murakawa et al. [6]. This system is described in Section 2.

The objective of the present work was to use this temperature stabilized system to study the influence of key parameters on the quality and growth rate of flame grown diamond layers. The parameters examined in this work are growth temperature, distance of the substrate from the cone of the inner flame, oxygen flow and acetylene supersaturation. For the experiments a relatively long deposition time of $1 \mathrm{~h}$ was used to minimize the effects of the initial diamond nucleation on the substrate as much as possible. Special attention was given to the homogeneity in thickness and quality of the deposited layers as these are considered essential for future applications of flame grown diamond.

\section{Experimental details}

After the first flame grown single-crystal diamonds were produced $[7,8]$ by our group, an improved experimental set-up was developed. A schematic overview of the arrangement is given in Fig. 1. A commercially available oxygen-acetylene welding torch fitted with a $1.0 \mathrm{~mm}$ orifice tip is used to generate the flame. The tip is cooled by a water-cooled copper rod to prevent

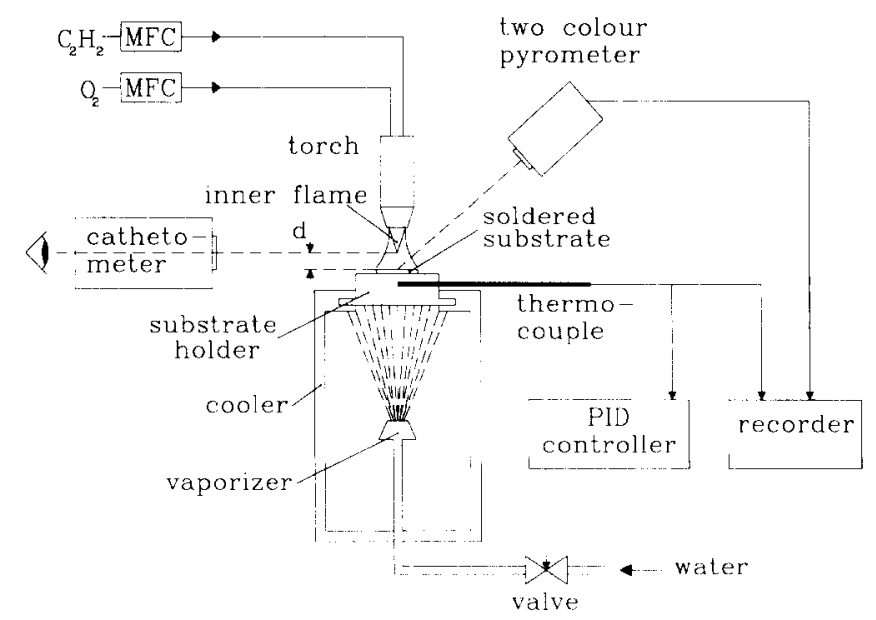

Fig. 1. Schematic overview of the flame deposition arrangement. 
ignition of the gas mixture inside the mixing chamber. Substrates are soldered onto a molybdenum substrate holder using a high temperature solder to ensure good thermal contact. The substrate holder is fitted on top of a stainless steel cooler supported with an $x-y-z$ translation stage for positioning the substrate relative to the torch. Inside the cooler a full-cone vaporizer is mounted to spray water against the back of the holder. A thermocouple fitted in the substrate holder probes the temperature approximately $2.5 \mathrm{~mm}$ beneath the centre of the growing surface. A temperature controller opens and closes the water valve of the vaporizer with a frequency of about $1 \mathrm{~Hz}$ depending on the thermocouple signal. In this way the temperature at this position in the holder can be regulated between $100^{\circ} \mathrm{C}$ and $1200^{\circ} \mathrm{C}$ with an accuracy of $1^{\circ} \mathrm{C}$. The actual growth temperature $T_{\mathrm{g}}$ is measured within $\pm 10^{\circ} \mathrm{C}$ relative $\left( \pm 20^{\circ} \mathrm{C}\right.$ absolute) using a two-colour IR pyrometer. Because of interference from the flame, $T_{\mathrm{g}}$ exhibits fast fluctuations which makes the pyrometer unsuitable for controlling the vaporizer. Both the thermocouple and pyrometer measurements are registered with a recorder. Depending on the growth conditions $T_{\mathrm{g}}$ is about $200^{\circ} \mathrm{C}$ higher than the installed substrate temperature at the position of the thermocouple. A cathetometer is used to measure and to provide precise control of the distance $d$ between the inner flame cone and the substrate surface within $\pm 0.1 \mathrm{~mm}$. The mass flows of the high purity source gases oxygen $(99.999 \%)$ and acetylene $(99.6 \%)$ are controlled within \pm 0.02 standard $1 \mathrm{~min}^{-1}(\mathrm{~s} / \mathrm{m})$. To prevent disturbance of the flame by ambient air [5], the torch and the cooler with the substrate are partly shielded by an exhaust hood. The exhaust ensures the safety of the researchers by removing combustion residuals, e.g. carbon monoxide. This set-up provides improved control of the growth parameters during extended experiments up to $11 \mathrm{~h}$ long [9]. The first results using the set-up have already been reported [10].

Square $1.2 \times 1.2 \mathrm{~cm}^{2}$ molybdenum substrates $0.5 \mathrm{~mm}$ thick were used in all experiments. Molybdenum substrates are easy to handle and are able to deal with the large thermal gradients which are inherent to flame deposition. Prior to deposition they are scratched with micrometre sized diamond powder to enhance diamond nucleation, cleaned with acetone and blown dry in air. The initial diamond nucleation is expected to vary between the different experiments, because the intensity at which the substrates are scratched with diamond powder is not quantified. To minimize the effect of these variations on the total deposit as much as possible, a deposition time of $1 \mathrm{~h}$ was used for all experiments.

The influence of the key parameters on the growth rate and the quality of the deposits was examined for a large number of experiments. These parameters are the growth temperature $T_{\mathrm{g}}$, the distance of the substrate from the inner flame cone $d$, the absolute oxygen flow $f_{\mathrm{ox}}$, and the acetylene supersaturation $S_{\mathrm{ac}}$. The ranges in which the mentioned parameters were varied are given in Table 1, as well as the conditions which were kept constant in the experiments. The table also refers to the figures in which the different sets of parameters are presented.

$S_{\mathrm{ac}}$ is defined as the acetylene flow minus the acetylene flow of a neutral flame divided by the latter, all with the same absolute oxygen flow. This definition is slightly different from that used earlier by our group $[10,11]$. The neutral flame, for which the acetylene feather has just disappeared, is determined by sight. The oxygen-toacetylene flow ratio of a neutral flame is known to depend on the total flow, the diameter of the orifice and the geometry of the burner. This means that in general there is an undefined zero point when the gas composition is described by the oxygen-to-acetylene flow ratio $R$ as is normally done. Therefore, the acetylene supersaturation as defined in this work is probably a better parameter to describe the gas composition. A disadvantage of the use of $S_{\mathrm{ac}}$ is the human factor in the determination of the neutral flame.

\section{Characterization}

The surface morphology of the grown layers was examined by optical microscopy and scanning electron microscopy (SEM). The layer thickness of the deposits was determined by focusing with an optical microscope. For this purpose a Nomarski differential interference contrast microscope was used. The relative position of an object in focus on the microscope stage can be determined within $\pm 0.1 \mathrm{~mm}$ in the horizontal plane and within $\pm 1 \mu \mathrm{m}$ in the vertical direction. Assuming that the substrates are flat, the vertical position of the molyb-

TABLE 1. Deposition conditions

Fixed deposition conditions for all experiments

Welding torch No. 1 , with a $\phi 1 \mathrm{~mm}$ orifice

Substrate $12 \times 12 \times 0.5 \mathrm{~mm}^{3}$ molybdenum

Deposition time $1 \mathrm{~h}$

Varied deposition conditions

\begin{tabular}{lcll}
\hline Parameter & Range & Fixed parameters & Figure \\
\hline$T_{\mathrm{g}}\left({ }^{\circ} \mathrm{C}\right)$ & $1010-1215$ & $d=1.0, S_{\mathrm{ac}}=6, f_{\mathrm{ox}}=1.4$ & 3 \\
$T_{\mathrm{g}}\left({ }^{\circ} \mathrm{C}\right)$ & $950-1230$ & $d=1.5, S_{\mathrm{ac}}=6, f_{\mathrm{ox}}=1.4$ & 3,4 \\
$d(\mathrm{~mm})$ & $0.4-1.4$ & $T_{\mathrm{g}}=1200, S_{\mathrm{ac}}=6, f_{\mathrm{ox}}=1.4$ & 5,6 \\
$S_{\mathrm{ac}}(\%)$ & $4-10$ & $T_{\mathrm{g}}=1200, d=1.0, f_{\mathrm{ox}}=1.4$ & 7,8 \\
$S_{\mathrm{ac}}(\%)$ & $2-8$ & $T_{\mathrm{g}}=1200, d=0.5, f_{\mathrm{ox}}=1.4$ & 7 \\
$f_{\mathrm{ox}}\left(\mathrm{standard}^{-1}\right)$ & $1.0-2.0$ & $T_{\mathrm{g}}=1200, d=1.0, S_{\mathrm{ac}}=6$ & 9 \\
1 min $\left.^{-1}\right)$ & & & \\
\hline
\end{tabular}


denum surface at any point in the horizontal plane can be calculated by determining the positions of the four non-covered corners of the substrate. At any point the thickness of the deposit is given by the difference between its surface position and the calculated position of the substrate in the vertical direction. Measurements performed in this way point out that, prior to growth, the substrates indeed are flat within $\pm 1 \mu \mathrm{m}$, probably because of the intensive polishing with diamond powder.

Raman spectroscopy was used to compare the relative quality of the deposited films. The Raman arrangement consists of a $100 \mathrm{~mW} \mathrm{Ar}{ }^{+}$ion laser as an excitation source, a triple monochromator used at a resolution of approximately $1 \mathrm{~cm}^{-1}$ and a photon-counting system for detection. Based on the Raman spectrum, a quality number $Q$ is introduced. $Q$ is defined by 1000 times the surface of the diamond peak divided by the surface of the background between $1100 \mathrm{~cm}^{-1}$ and $1700 \mathrm{~cm}^{-1}$ (see Fig. 2(a)). The relation between $Q$ and a number of Raman spectra is presented in Fig. 2(b). Based on repeated measurements of the same sample, the experimental error in the determination of $Q$ is expected to be $\pm 10 \%$. The quality of a single diamond crystallite is normally judged by the full width at half-maximum (FWHM) of the diamond peak $\left(1332 \mathrm{~cm}^{-1}\right)$ in the Raman spectrum. However, polycrystalline films consist of diamond crystallites in a matrix of amorphous carbon and crystalline graphite. In the course of our investigations Raman spectra like spectrum d in Fig. 2 were observed, with a smaller FWHM than spectrum $c$ in the same figure. The presence of a small number of high quality diamond crystallites in a low quality matrix is probably responsible for this Raman signature. Therefore the quality number as defined in the present work is in
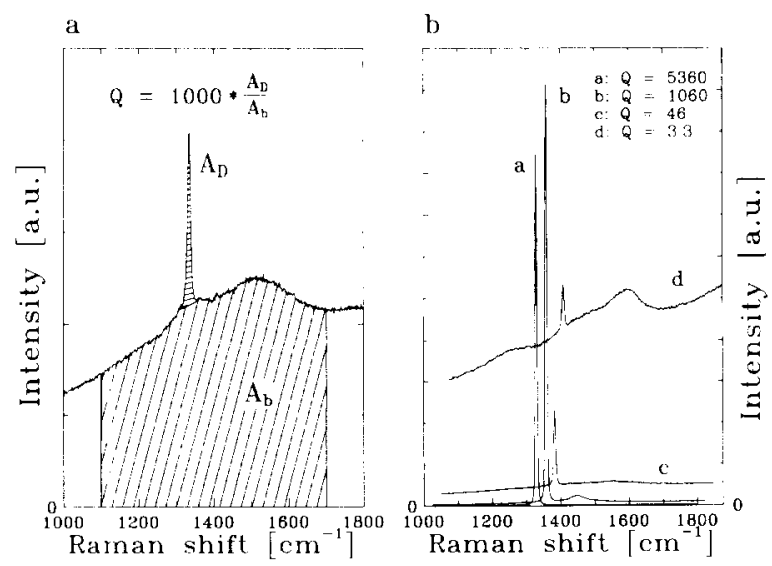

Fig. 2. Raman spectra illustrating the definition of $Q$ (a) and values of $Q$ for different spectra (b). The spectra in part $b$ represent natural diamond type IIa (a), flame-grown single-crystalline diamond (b) and polycrystalline diamond layers (c and d). For clarity spectra b, c and $\mathrm{d}$ are shifted to the right-hand side by $25 \mathrm{~cm}^{-1}, 50 \mathrm{~cm}^{-1}$ and $75 \mathrm{~cm}^{-1}$ respectively. our opinion a better way of quantifying the overall quality of the deposits.

\section{Results}

After growth, most of the deposits exhibit the following features when inspected by optical and scanning electron microscopies. The layers are almost circularly symmetric with respect to the flame axis, as was to be expected from a geometrical point of view. The central area of the deposits shows a continuous polycrystalline diamond film with $\{111\}$ faces at random orientations (octahedral growth). This area with a diameter of about $3 \mathrm{~mm}$, is uniform in thickness (within $\pm 10 \%$ ) and homogeneous in morphology (see also Figs. 6(b) and 6(c)). It is surrounded by a ring of enhanced growth and roughness which is about $0.6 \mathrm{~mm}$ wide. This ring consists of columnar grown individual crystallites showing $\{100\}$ faces more or less parallel to the substrate. Outside this ring little or no growth occurs. The central area is well attached to the molybdenum substrate, scratching with diamond powder generally has little effect on this part of the deposit. However, the individual crystallites in the surrounding ring are easily polished away. Unless stated otherwise, all the samples discussed in this section exhibit the features described above. From the previous description it is evident that only the central area of the flame grown deposits are of practical use in future applications. Therefore the growth rates and qualities presented in this paper only concern this region.

The growth rate $v_{\mathrm{g}}$ as a function of the growth temperature for two series of experiments with a different distance $d$, is given in Fig. 3(a). The fixed values of the other parameters are given in Table 1 . The habit of the diamond crystallites in the films grown at a distance of $1.5 \mathrm{~mm}$ changes from octahedral at low temperatures,
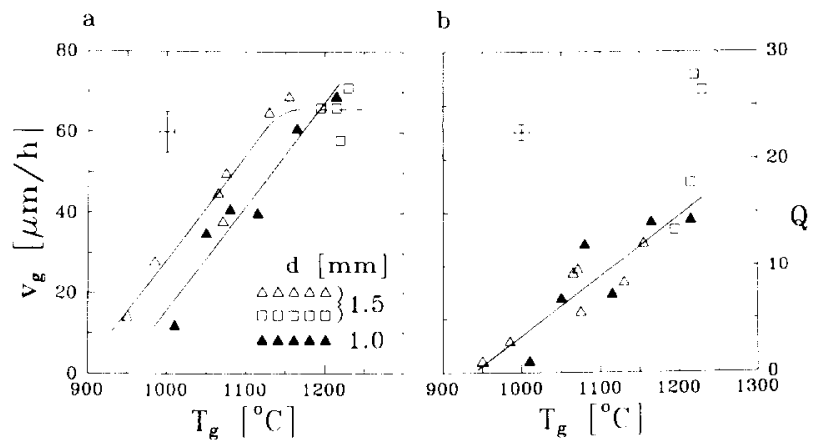

Fig. 3. Growth rate (a) and quality (b) of fiame grown diamond films as a function of the growth temperature at two different distances. The symbols indicate whether the crystallites in the deposits show $\{111\}$ faces (triangles) or $\{100$; faces (squares\}. The lines are guides to the eyes and indicate whether the central area of the deposit forms a continuous layer (continuous) or consists of separate crystallites (dashed). The average error in the data is indicated by the error bars. 
via cubo-octahedral (both $\{111\}$ and $\{100\}$ faces) at $T_{\mathrm{g}} \approx 1130^{\circ} \mathrm{C}$, to columnar growth at higher temperatures (Fig. 4). No such change is observed for samples grown
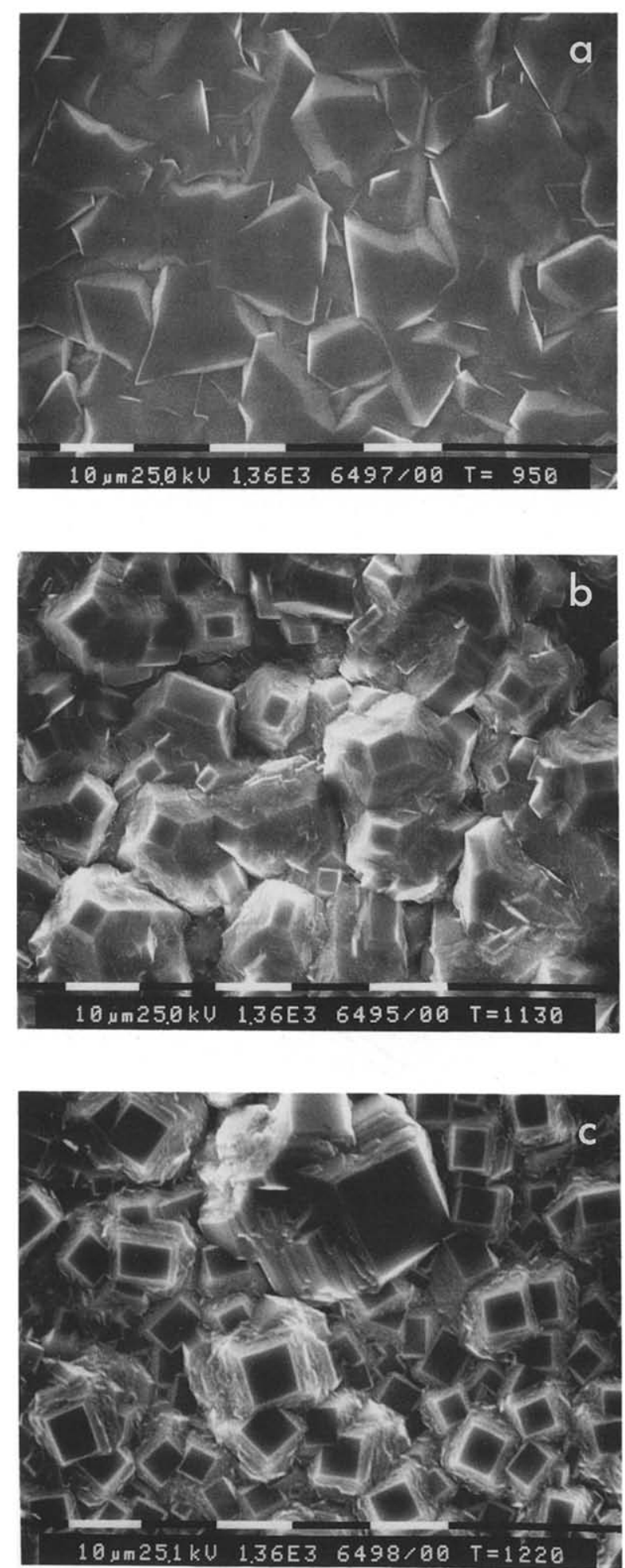

Fig. 4. SEM images of the central area of diamond films grown at a distance of $1.5 \mathrm{~mm}$ with $T_{\mathrm{g}} \approx 950^{\circ} \mathrm{C}$ (a), $T_{\mathrm{g}} \approx 1130^{\circ} \mathrm{C}$ (b) and $T_{\mathrm{g}} \approx 1220^{\circ} \mathrm{C}$ (c). at $1 \mathrm{~mm}$. The change in crystal habit with temperature has also been reported by other authors [12, 13]. Excluding data obtained from films with a different crystal habit, both series show linear behaviour of the growth rate with $T_{\mathrm{g}}$ within the examined temperature range. Although the data are somewhat more scattered, there is also a roughly linear relation between the quality of the deposits and the growth temperature (Fig. 3(b)). Deposits grown at temperatures above $1230^{\circ} \mathrm{C}$ fractured and sputtered from the substrate while cooling down at the end of the experiment. The foregoing leads to the conclusion that the best results are obtained at $T_{\mathrm{g}} \approx 1200^{\circ} \mathrm{C}$ and $d<1.5 \mathrm{~mm}$.

Both the growth rate and the quality of the diamond films increase with decreasing distances (Fig. 5). For distances above $1.0 \mathrm{~mm}$ the quality shows a tendency to stabilize. Based on these results the preferred distance for diamond growth is as close as possible to the flame core. However, at $d<0.8 \mathrm{~mm}$ the centre of the diamond layer breaks up in separate crystallites as can be seen in Fig. 6(a). This makes the deposits unsuitable for practical applications. Therefore a distance of $1.0 \mathrm{~mm}$ (to be on the safe side) is considered to be the most appropriate under these circumstances (Figs. 6(b) and 6(c)).

The growth rate and the quality of diamond deposits as a function of the acetylene supersaturation is given in Fig. 7. Diamond growth appears to be extremely sensitive to this parameter in a number of ways. At supersaturations below $8 \%$, the growth rate is approximately linear with $S_{\mathrm{ac}}$. Except for extremely low supersaturations the quality in this range (SEM image shown in Fig. 8(a)) appears to be quite indifferent to variations in $S_{\text {ac. }}$ At high supersaturations the central area breaks up into large separate crystallites. These are still octahedrally shaped at $S_{\mathrm{ac}}=8 \%$ (Fig. $8(\mathrm{~b})$ ) but have a cubic signature at $S_{\mathrm{ac}}=10 \%$ (Fig. 8(c)). With a supersaturation of $2 \%$ the intersection of the acetylene feather with the substrate and with this the central area of the deposit vanishes at a distance of $1.0 \mathrm{~mm}$. At a distance of $0.5 \mathrm{~mm}$,
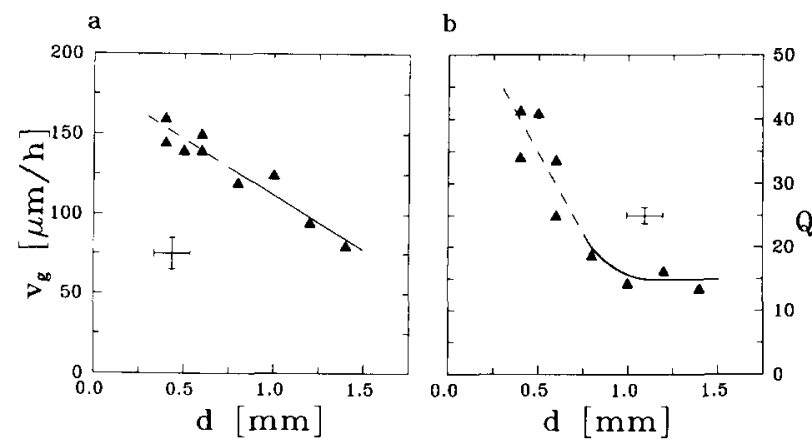

Fig. 5. Growth rate (a) and quality (b) of diamond deposits as a function of the distance of the substrate from the cone of the inner flame. The shapes of the lines and markers indicate the same features as stated in Fig. 3. 

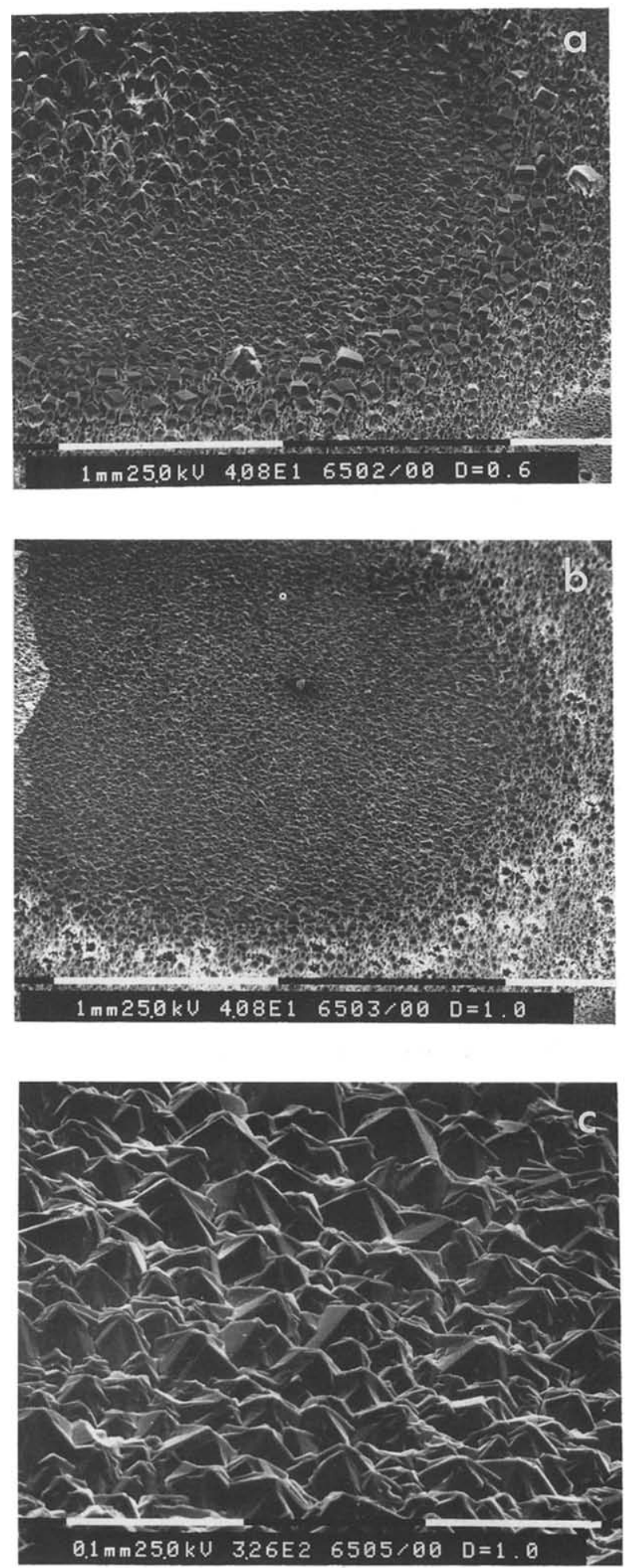

Fig. 6. SEM images of diamond films grown at a distance of $0.6 \mathrm{~mm}$ (a) and $1.0 \mathrm{~mm}(\mathrm{~b}$ and $\mathrm{c})$. Images $\mathrm{a}$ and $\mathrm{b}$ show somewhat more than one quadrant of the diamond films. The centre of the films is shown in the upper left-hand corner and the circle of enhanced growth along the bottom and right-hand side of the pictures. Photograph $\mathrm{c}$ shows the central area of $b$ in more detail. All photographs were taken at an angle of about 35 with the normal of the surface.

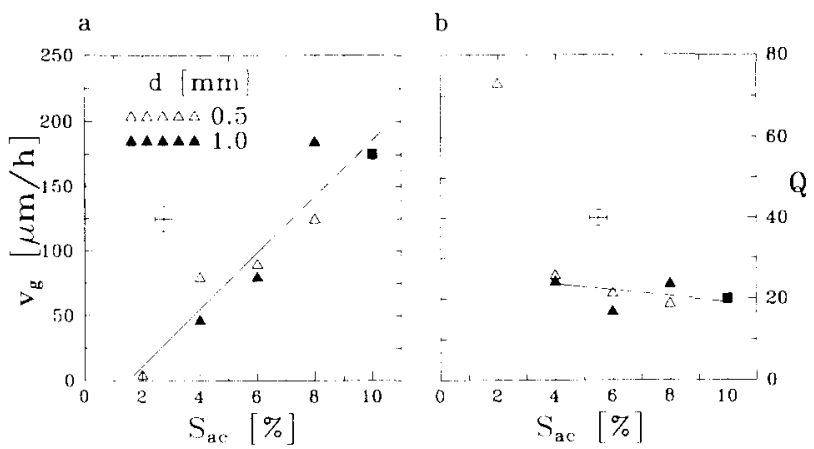

Fig. 7. Growth rate (a) and quality (b) of diamond deposits as a function of the acetylene supersaturation at two different distances. The shapes of the lines and markers indicate the same features as stated in Fig. 3.

however, a continuous layer with a diameter of $1 \mathrm{~mm}$ could be grown. This sample exhibits an extremely low growth rate but also an extremely high quality (see Fig. 7). In order to obtain a normal central area with a high growth rate a supersaturation of $6 \%$ appears optimal under the given conditions.

In Fig. 9 the growth rate and the quality are given as a function of the absolute oxygen flow keeping $S_{\mathrm{ac}}$ constant. Both quantities vary linearly with respect to this parameter. High quality films obtained at high flow rates have also been reported by Snail and Craigie [14]. At low oxygen flows, the morphology of the diamond films is changed from octahedral to cubo-octahedral. However, the number of experiments performed is not large enough to determine whether this introduces a discontinuity in the relation between the growth rate and the oxygen flow or not. The flow of 2.0 standard $1 \mathrm{~min}^{-1}$ is the largest oxygen flow possible in our set-up using a orifice of $1.0 \mathrm{~mm}$.

\section{Discussion}

With the experimental set-up as described in Section 2, diamond films with a diameter of $3 \mathrm{~mm}$ which are uniform in thickness and morphology are obtained. In experiments performed with a larger flame $(1.6 \mathrm{~mm}$ diameter orifice), homogeneous films of $7 \mathrm{~mm}$ diameter have already been grown [9]. This indicates that the temperature gradients in the radial direction along the growing surface are less than expected. Owing to the large thermal conductivity of the diamond this holds especially after the deposition of the first continuous layer. There is little information on the spatial variations of the gas phase composition in the radial direction along the substrate. In situ techniques for examining the gas phase, such as laser induced fluorescence, must give an answer in the future.

A clear relationship has been found between the 

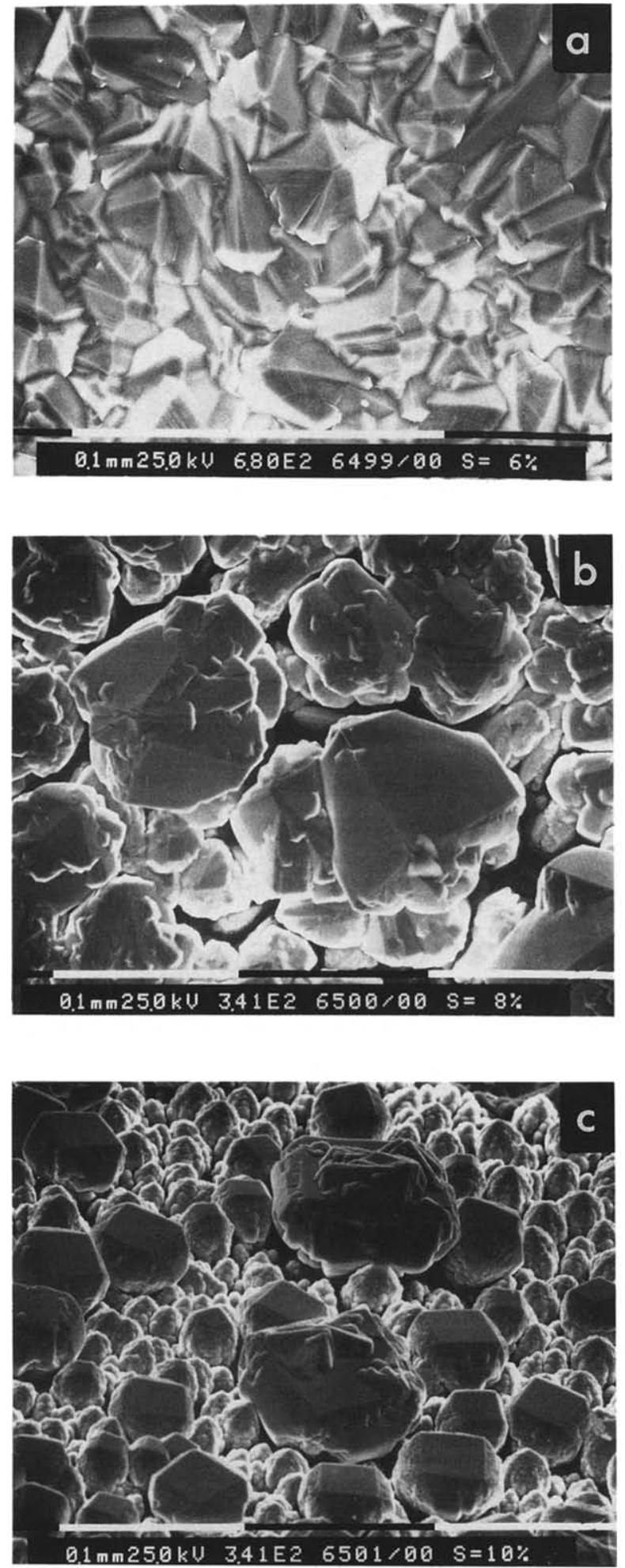

Fig. 8. SEM images showing details in the central area of diamond deposits grown with a supersaturation of $6 \%(a), 8 \%$ (b) and $10 \%$ (c) respectively. Photograph $\mathrm{c}$ was taken at an angle of about $35^{\circ}$ with the normal of the surface to show the shape of crystallites with $\{100\}$ top faces.
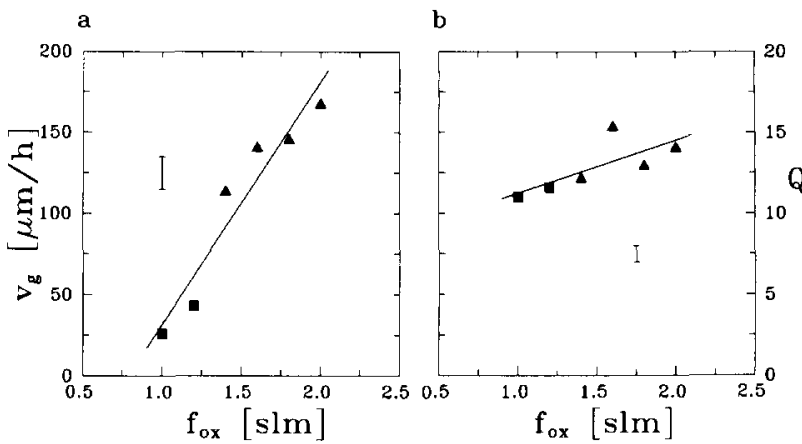

Fig. 9. Growth rate (a) and quality (b) of diamond films as a function of oxygen flow. The shapes of the lines and markers indicate the same features as stated in Fig. 3.

growth rate and each of the investigated parameters. The relationship between the quality of the diamond and these parameters is somewhat less pronounced. It should be noted at this point that, apart from the influence of the examined parameter, the quality is also dependent on the growth rate. When the influence of other parameters is excluded, in general crystal quality is known to decrease for increasing growth rates in numerous crystal growth processes. The best conditions for growing continuous, polycrystalline diamond films at high growth rates $\left(v_{\mathrm{g}}=168 \mu \mathrm{m} \mathrm{h}^{-1}\right)$ with our equipment appear to be $T_{\mathrm{g}}=1200^{\circ} \mathrm{C}, d=1 \mathrm{~mm}, S_{\mathrm{ac}}=6 \%$ and $f_{\mathrm{ox}}=2.0$ standard $1 \mathrm{~min}^{-1}$. However, most of the experiments were done using an oxygen flow of 1.4 standard $1 \min ^{-1}$.

When the results of experiments within one series are compared they are consistent within the experimental error. This indicates that the influence of the initial nucleation on the molybdenum on the total deposit is negligible, as was intended by the use of growth times of $1 \mathrm{~h}$. However, the absolute values of the presented growth rates and qualities should be regarded with some care when different series of experiments are compared with each other. A point representing growth at $T_{\mathrm{g}}=$ $1200^{\circ} \mathrm{C}, d=1.0 \mathrm{~mm}, S_{\mathrm{ac}}=6 \%$ and $f_{\mathrm{ox}}=1.4$ standard $1 \mathrm{~min}^{-1}$, can be found in each of the presented diagrams. The growth rate at these points ranges from $68 \mu \mathrm{m} \mathrm{h}^{-1}$ to $113 \mu \mathrm{m} \mathrm{h}^{-1}$ and the quality ranges from 12.5 to 22 . Those ranges are much larger than expected from errors in each of the independent series of experiments. A possible cause of this effect might be the presence of acetone vapour in the gas flow. The acetone originates from the acetylene bottle in which the acetylene is dissolved in acetone to stabilize it. When the acetylene pressure drops during the use of a bottle, the acetone content in the gas flow will increase because the partial vapour pressure of acetone remains constant. Deposits grown from a full bottle show higher growth rates and lower qualities compared with deposits grown under the same circumstances from an almost empty bottle. For 
example, $v_{\mathrm{g}}=65 \mu \mathrm{m} \mathrm{h}^{-1}$ and $Q=6$ vs. $v_{\mathrm{g}}=35 \mu \mathrm{m} \mathrm{h}^{-1}$ and $Q=22$ for samples grown at $T_{\mathrm{g}}=1100^{\circ} \mathrm{C}, d=$ $1.4 \mathrm{~mm}, S_{\mathrm{ac}}=6 \%$ and $f_{\mathrm{ox}}=1.4$ standard $1 \mathrm{~min}^{-1}$. Part of this problem has been solved by using less than the first $25 \%$ of a new bottle to perform each of the presented series of experiments. However, variations in the impurity content of the different acetylene bottles cannot be excluded as the main reason for the observed differences between the individual series. Unfortunately, this cannot be proved because in the present set-up the gas composition cannot be monitored. To solve this problem in the future the acetone could be removed by passing the acetylene through an activated charcoal trap and by monitoring the gas composition [5].

\section{Conclusions}

An experimental set-up has been developed which provides good control of the growth parameters, especially the deposition temperature and the substrate flame core distance, during long-term flame deposition experiments. With this equipment diamond layers of good quality and uniformity in thickness and morphology with a diameter of approximately $3 \mathrm{~mm}$ have been grown for a wide range of process conditions. Experiments with a larger flame show that even homogeneous films with $7 \mathrm{~mm}$ diameter can be grown. The present set-up can still be further improved by adding an activated charcoal trap to the gas system in order to remove acetone from the acetylene flow.

Both the growth rate and the quality of the diamond films are strongly dependent on all the investigated parameters. The largest growth rate $\left(v_{\mathrm{g}}=168 \mu \mathrm{m} \mathrm{h}^{-1}\right)$ with a reasonable quality $(Q=14)$ was reached at $T_{\mathrm{g}}=$ $1200{ }^{\circ} \mathrm{C}, d=1.0 \mathrm{~mm}, S_{\mathrm{ac}}=6 \%$ and $f_{\mathrm{ox}}=2.0$ standard $1 \min ^{-1}$. The highest overall quality $(Q=70)$ was found at $T_{\mathrm{g}}=1200^{\circ} \mathrm{C}, d=0.5 \mathrm{~mm}, S_{\mathrm{ac}}=2 \%$ and $f_{\mathrm{ox}}=1.4 \mathrm{stan}-$ dard $1 \mathrm{~min}^{-1}$, however, here the central area is small $(\phi$ $1 \mathrm{~mm})$ and the growth rate is extremely low $\left(v_{\mathrm{g}}=4 \mu \mathrm{m}\right.$ $\mathrm{h}^{-1}$ ). An even better quality is to be expected at $f_{\mathrm{ox}}=2.0$ standard $1 \mathrm{~min}^{-1}$. Besides the growth rate and the quality, the preferred crystal habit of the films also appears to be affected by the investigated parameters.
The present parametric study of polycrystalline diamond growth forms a solid base for future experiments on the growth of large size, high quality single crystals by flame deposition [9].

\section{Acknowledgments}

The authors wish to thank Ing. W. Vollenberg for his help in recording Raman spectra, while the technicians at Drukker International are acknowledged for their assistance in the development of the experimental setup. Further they acknowledge the Netherlands Technology Foundation (STW) and the Netherlands Organization for Scientific Research (NWO) for partial financial support.

\section{References}

1 Y. Hirose and N. Kondo, Extended Abstracts, 35th Jpn. Appl. Phys. Spring Meet., March 1988, p. 434.

2 Y. Hirose, S. Amanuma and K. Komaki, J. Appl. Phys., 68 (1990) 6401 .

3 L. M. Hanssen, W. A. Carrington, J. E. Butler and K. A. Snail, Mater. Lett., 7 (1988) 289.

4 L. M. Hanssen, K. A. Snail, W. A. Carrington, J. E. Butler, S. Kellogg and D. B. Oakes, Thin Solid Films, 196 (1991) 271.

5 D. B. Oakes, J. E. Butler, K. A. Snail, W. A. Carrington and L. M. Hanssen, J. Appl. Phys., 69 (1991) 2602.

6 M. Murakawa, S. Takeuchi and Y. Hirose, Surf. Coat. Technol., $43-44(1990) 22$

7 G. Janssen, W. J. P. van Enckevort, J. J. D. Schaminée, W. Vollenberg, L. J. Giling and M. Seal, J. Cryst. Growth, 104 (1990) 752.

8 G. Janssen, W. Vollenberg, L. J. Giling, W. J. P. van Enckevort, J. J. D. Schaminée and M. Seal, Surf. Coat. Technol., 47 (1990) 113.

9 J. J. Schermer and G. Janssen, unpublished results, 1992.

10 G. Janssen, J. J. Schermer, W. J. P. van Enckevort and L. J. Giling, J. Cryst. Growth, 125 (1992) 42.

11 G. Janssen, W. J. P. van Enckevort, W. Vollenberg and L. J. Giling, Diamond Relat. Mater., I (1992) 789

12 R. Komanduri, K. S. Snail and L. L. Fehrenbacher, Philos. Mag. Lett., 62 (1990) 283.

13 K. V. Ravi, J. Mater. Res., 7 (1992) 384.

14 K. A. Snail and C. J. Craigie, Appl. Phys. Lett., 58 (1991) 1875. 